\title{
Ronald Spores y los años tempranos en la Mixteca: una semblanza
}

\author{
Marcus Winter
}

4 n la primavera de 1965 iba a terminar mi licenciatura en artes liberales y no tenía planes para el futuro. Un amigo, Bruce Bourque, nativo de Nueva Inglaterra, estudiaba en la Universidad de Massachusetts en el mismo pueblo que mi escuela, Amherst College. Un día me contó que su enfoque era la antropología y que pensaba hacer un posgrado con especialidad en arqueología para después ejercer la profesión de arqueólogo. Desde chico me interesaba la arqueología y había participado durante dos veranos en expediciones al suroeste de Estados Unidos con arqueólogos del Museo de Historia Natural de Chicago. Dado que el Amherst College no ofrecía cursos en arqueología, yo había optado por otras materias. Bruce me platicó que en su universidad habían anunciado un nuevo curso sobre arqueología con un profesor joven; sugirió que me inscribiera, ya que un acuerdo entre las escuelas permitía a los alumnos cursar materias en las otras instituciones. Me pareció una buena idea y me anoté en la lista. El primer día de clases conocí al profesor: era Ronald Spores.
Creo que Ron había terminado recientemente sus cursos del doctorado en Harvard y estaba en el proceso de terminar su tesis sobre la Mixteca o de finalizar las revisiones del manuscrito publicado en 1967 como The Mixtec Kings and their People. Pienso además que el año académico 1964-1965 fue su primer empleo como profesor. El curso se llamaba algo así como "Introducción a las civilizaciones del mundo antiguo”. Ron coordinó la clase; cada alumno tuvo que escoger un área del mundo y presentar una síntesis arqueológica. Para mí, la clase fue un gran éxito: inmediatamente despertó mi pasión dormida y nunca jamás dudé de cuál iba a ser mi carrera.

Terminó el año académico. Bruce se preparó para entrar a la Universidad de Colorado, donde lo aceptaron en el nivel de posgrado. (Llegó a ser un gran experto en la arqueología del noreste de Estados Unidos, especialista en el estado de Maine.) Ron se mudó a Nashville, en el estado de Tennessee, pues acababa de ser contratado como profesor en el Departamento de Sociología y Antropología de la Universidad Vanderbilt. Yo me fui a Arizona; durante el verano participé en un programa del Museo

\section{Ronald Spores and the Early Years in the Mixteca: a Profile}

MARCUS WINTER: Centro Instituto Nacional de Antropología e Historia-Oaxaca, México. winteroax@prodigy.net.mx 
de Historia Natural de Chicago y en septiembre entré a la Universidad de Arizona en Tucson para iniciar estudios de posgrado en el Departamento de Antropología.

En el transcurso de ese año me puse nuevamente en contacto con Ron para pedirle consejos, ya que quería participar en algún trabajo de campo durante el verano de 1966. Ron había conseguido permiso del Instituto Nacional de Antropología e Historia (INAH) para hacer un recorrido de superficie en el Valle de Nochixtlán en Oaxaca, México. Quería investigar y documentar los antecedentes de los mixtecos del periodo colonial que estudió para su tesis doctoral. Los documentos eran de especial interés, sobre todo el testamento de don Domingo de Guzmán, cacique de Yanhuitlán. Ron me invitó a participar como voluntario.

Al terminar el semestre nos encontramos en la ciudad de México. Nos quedamos una noche en el hotel Sevilla, que da a la calle de Serapio Rendón y que tenía un estacionamiento para su camioneta roja y blanca. (Por falta de imaginación, o por el gusto a lo conocido, durante los siguientes veinte años o más, al estar en la ciudad de México siempre me quedaba en el Sevilla o en el Compostela, frente al Sevilla, igual que Ron, Bill Autry y otros amigos y colegas.) Al día siguiente partimos por la carretera Panamericana hacia Oaxaca. En la tarde cruzamos la línea estatal de Puebla y llegamos a Huajuapan, donde pernoctamos en el hotel Laredo, otro de nuestros favoritos. Además de la buena comida, lo más llamativo fue la numerosa parvada de pájaros que durmió en un árbol del patio y que se encargó de despertar a los huéspedes con un ruido estruendoso al amanecer. No eran aves tropicales exóticas, sino gorriones corrientes. También a ellos —o más bien a sus descendientes — les falta imaginación, ya que todavía frecuentan el mismo árbol.

Ron me quería mostrar un sitio arqueológico en Huajuapan. Nos levantamos temprano y caminamos hacia el norte, subiendo una loma hasta llegar a una casa aislada donde platicamos con un señor y éste nos mostró unos pequeños cajetes de color anaranjado con base anular. Ya estábamos en el sitio. Subimos un poco más hasta la cima del cerro y vimos los montículos: unos pisos de estuco blanco expuestos y el panorama del valle de Huajuapan con la ciudad en el fondo junto al río Mixteco. Así conocí el primero de muchos sitios de Oaxaca que visitaría con Ron. Era el Cerro de las Minas, el centro ñuiñe, donde décadas más tarde realizaría varias temporadas de exploración. El señor era Liborio Viñas; él y su hermano eran los "dueños" del Cerro de las Minas. Años después, don Liborio nos ayudó con los permisos para la exploración y con la protección del sitio. Encontraríamos numerosos cajetes similares, ya que son las vasijas más típicas de la cultura ñuiñe.

Después del desayuno salimos de Huajuapan y más tarde, ese mismo, día llegamos a Nochixtlán y al restaurante-hotel del señor Rogelio Ballesteros y su esposa. Situado enfrente de la gasolinera, sería nuestro centro de alojamiento y operaciones. Llegaron de Nashville otros dos voluntarios: Cherie White, hija de unos amigos de Ron, y John Broster, estudiante en Vanderbilt, y ya estábamos listos para empezar el recorrido. Cada día salíamos temprano, estacionábamos el vehículo y caminábamos, acompañados por el sol mixteco y frecuentemente por la lluvia de la tarde y el lodo rojizo. Visitamos numerosos pueblos y registramos decenas de sitios, incluyendo los centros impresionantes de Jaltepec, Yucuñudahui, Cerro Jazmín, Tillo y otros dos - Etlatongo y Yucuitadonde diez años después tendría la oportunidad de excavar.

Becado por el gobierno de Estados Unidos después de su servicio militar, Ron había estudiado antropología con John Paddock en el Mexico City College. Además, al igual que otros futuros especialistas, como Donald Brockington y Kent V. Flannery, Ron tuvo su primera experiencia arqueológica en Oaxaca como participante en las prácticas de campo que organizó y dirigió Paddock en Yagul. El entusiasmo de Ron impulsó nuestro trabajo en Nochixtlán; él tenía una idea de lo que podía existir en el valle, mientras que nosotros, los estudiantes, nunca habíamos visto sitios tan inmensos y con tanta cerámica y lítica en la superficie. Ron describía cada sitio mientras que nosotros nos dedicábamos a buscar cerámica diagnóstica y artefactos. Cargamos las muestras de tepalcates en tenates hechos de palma para tortillas, atados con mecates que nos lastimaron los hombros. No llevábamos comida ni agua, pero al regresar al hotel nos dedicamos a reponer líquidos. John Broster fue quien batió el récord 


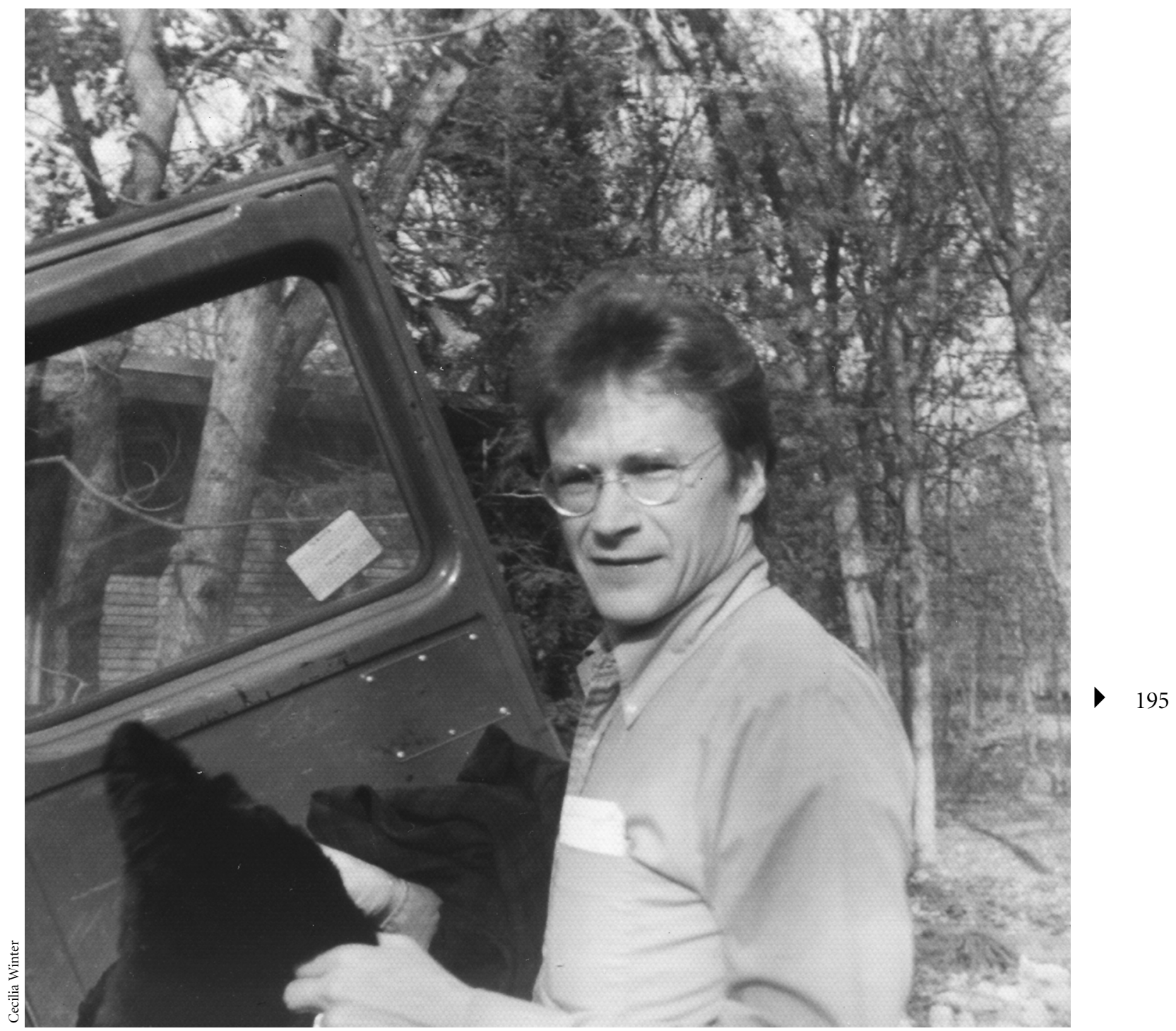

Ronald Spore, Nashville, Tennessee, 1971.

de la temporada: trece refrescos tomados en una tarde, la mayoría de ellos Barrilitos de varios colores.

A veces encontrábamos huesos fosilizados en los arroyos y yo esperaba localizar un sitio paleo-indio, como los del valle de San Pedro, cerca de Tucson. En las afueras de Nochixtlán hallamos la mandíbula de un mamut y un raspador de sílex en la superficie cercana. Cuando terminó la temporada, Ron reportó el hallazgo a José Luis
Lorenzo, jefe del Departamento de Prehistoria del INAH en la ciudad de México y parece que Lorenzo visitó el sitio. El hueso estaba en una matriz de sedimento rojo y tenía que ser pre-pleistocénico. En 1972 Ron publicó el recuento del recorrido, intitulado An Archaeological Settlement Survey of the Nochixtlan Valley, Oaxaca, primer número de la serie de monografías que sobre diversos temas de Oaxaca editaron las Publicaciones en Antropo- 


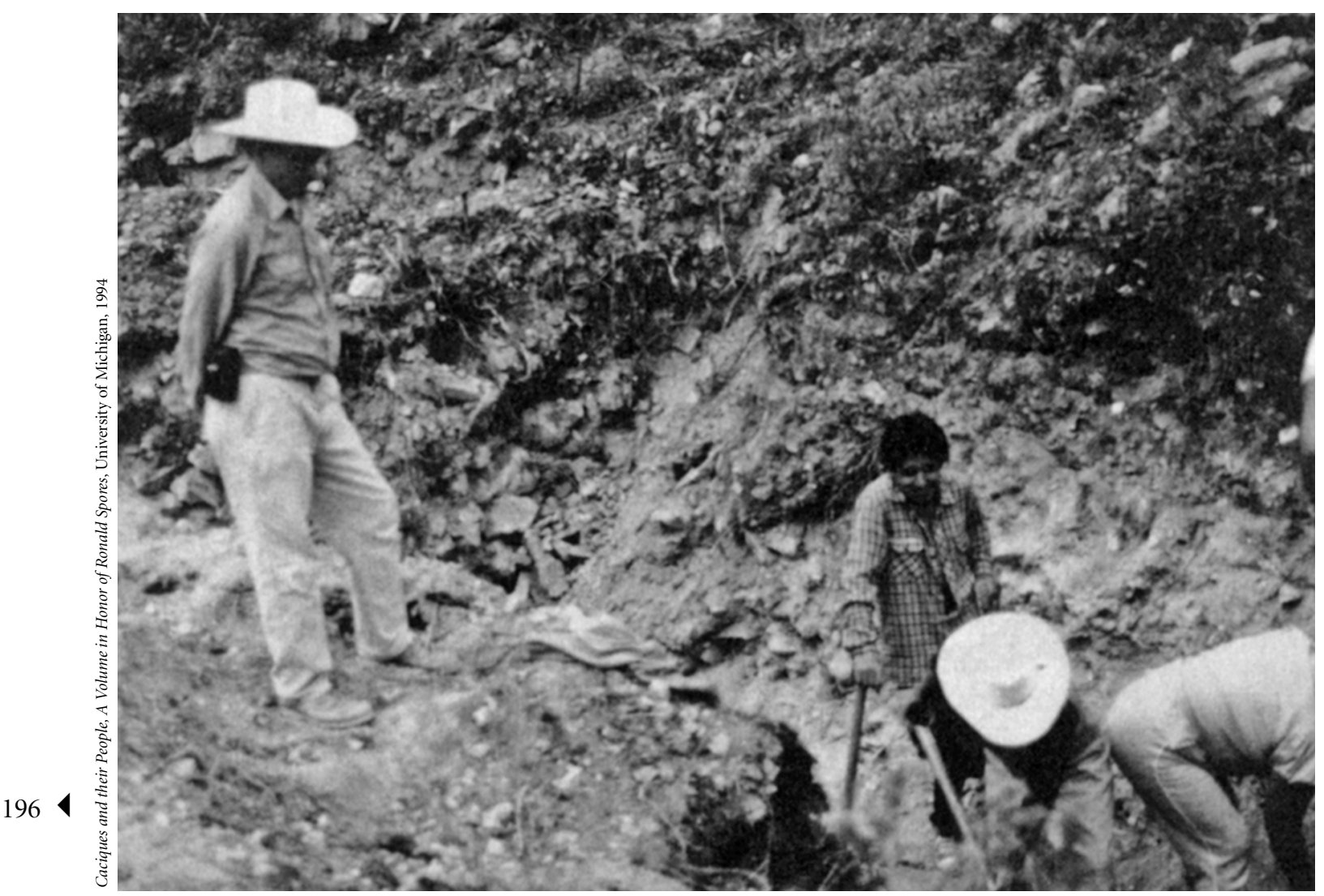

Ronald Spores en una excavación sobre la Mixteca prehistórica.

logía de la Universidad de Vanderbilt (VUPAs, por sus siglas en inglés).

Ron es una persona gregaria y amable, y siempre establece contactos personales; ese verano, gracias a él, conocimos a mucha gente. Nos visitó el escritor Ross Parmenter, autor de $A$ Week in Yanhuitlán y otras obras. Los fines de semana íbamos a veces a Mitla a quedarnos en la posada La Sorpresa, donde John Paddock tenía el laboratorio arqueológico de su Instituto de Estudios Oaxaqueños. En el mismo edificio estaba el Museo Frissell de Arte Zapoteca con piezas arqueológicas inigualables de todas partes del estado de Oaxaca. Ahí también vivía el artista Howard Leigh, con su propia colección de piezas zapotecas, y Darío Quero, administrador de la posada, quien tuvo una colección de adornos de jadeíta. Además, Kent V. Flannery, entonces del Instituto Smithsoniano en Washington, y sus colaboradores y estudiantes estaban alojados en la posada. El año anterior Kent había iniciado su proyecto "Prehistoria y ecología humana en el valle de Oaxaca", y en 1966 estaban con él Anne y Michael Kirkby, geógrafos de Inglaterra; Aubrey Williams, etnógrafo de la Universidad de Maryland; James A. Neely, arqueólogo que había trabajado con Flannery en el Medio Oriente y que estaba investigando el lugar llamado Hierve el Agua; Christopher L. Moser, fotógrafo y estudiante de arqueología, y Richard Orlandini, también estudiante de arqueología. Entre los oriundos de Mitla y principales ayudantes de Flannery estaban Eligio Martínez, Ernesto Martínez y Pablo García; los dos últimos posteriormente consiguieron empleo en el INAH. El contacto con Kent condujo a una colaboración entre los proyectos; para mí resultó valioso, ya que me ofreció trabajo para la temporada siguiente. 


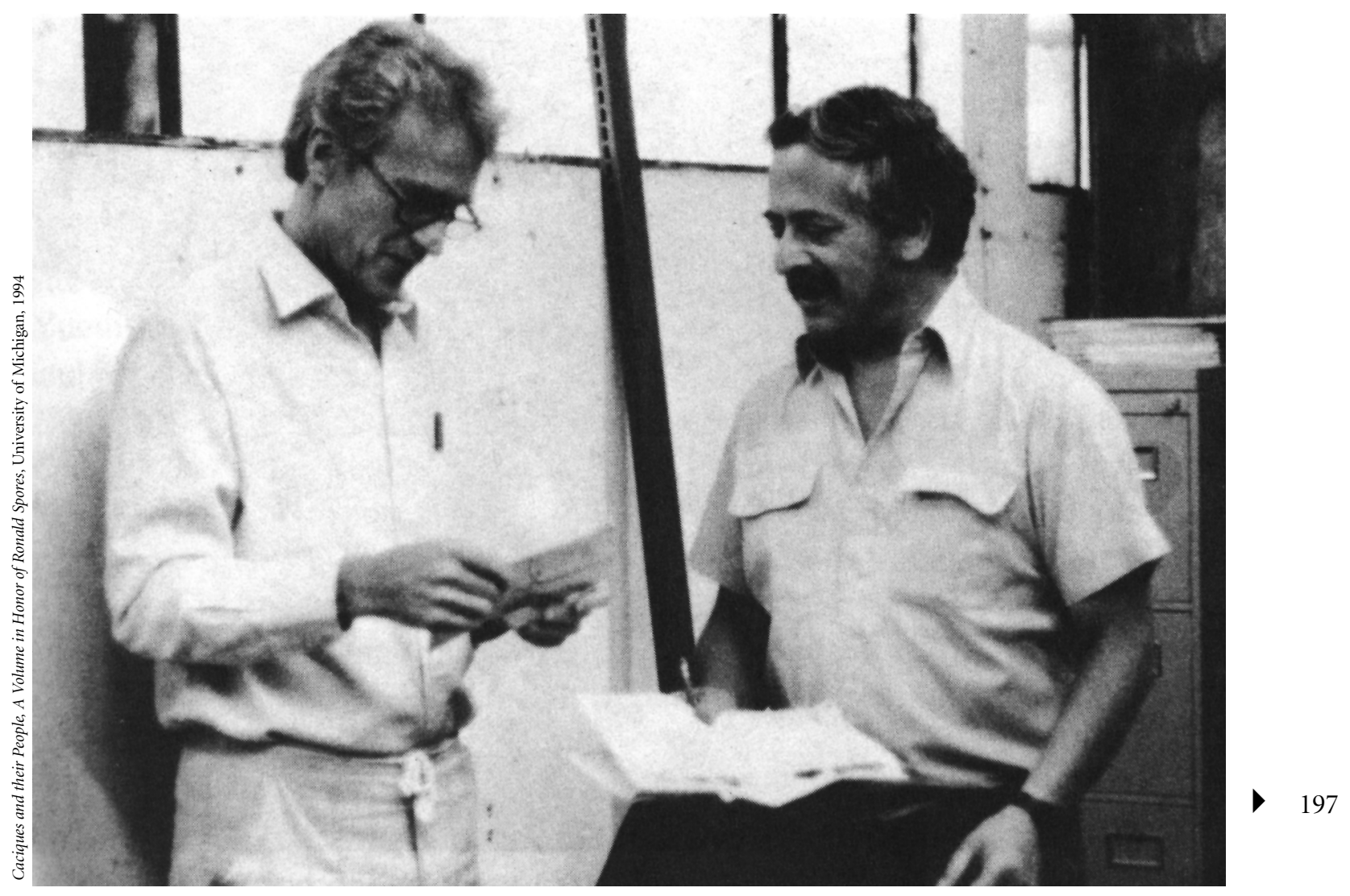

Ronald Spores y Manuel Esparza, director de los archivos de Oaxaca.

Una de las amistades más significativas en la vida de Ron empezó una tarde que fuimos a Yucuita por primera vez. Antes de llegar al pueblo, un señor que trabajaba en su campo nos hizo señas y vino corriendo hacia la camioneta. Se llamaba Gildardo Ramos — posteriormente inmortalizado en la secuencia cronológica (fases Cruz y Ramos) y la tipología cerámica (Gildardo Lustrous Red) del valle de Nochixtlán-. Ron platicó con él sobre varios temas, incluyendo el de los sitios arqueológicos existentes en el área. Tiempo después, Ron lo llevó a Nashville, donde trabajó como jardinero para unas personas acomodadas; al principio, Gil dormía clandestinamente debajo de una mesa en las oficinas del Departamento de Antropología. Así comenzó una fuga de familiares y amigos de Gil hasta llegar al punto en que probablemente había más Cruces y Ramos en Nashville que en Yucuita. Una vez le pregunté a Gil cómo le había ido, y me contó que se había enfadado con la comida, ya que lo único que sabía pedir en los restaurantes era "jámonegs".

En 1967 Ron volvió al valle de Nochixtlán, excavó pozos estratigráficos en Yucuita, Chachoapan y Yanhuitlán, y obtuvo datos para establecer una secuencia cronológica. Publicó estos trabajos en 1974 como la VUPA núm. 11, Stratigraphic Excavations in the Nochixtlán Valley, Oaxaca. En 1970 Ron emprendió el Proyecto del Valle de Nochixtlán impulsado por la Fundación Nacional de Ciencia de Estados Unidos. John Broster y Michael D. Lind fueron sus ayudantes principales; excavaron en Yucuita, Yucuñudahui y Chachoapan. Lind supervisó la exploración de un palacio del Posclásico en Chachoapan, que sirvió como base para su tesis doctoral en la Universidad de Arizona. Los datos fueron publicados en dos monogra- 
fías por Lind: Postclassic and Early Colonial Mixtec Houses in the Nochixtlan Valley (VUPA 23, 1979) y The Sociocultural Dimensions of Mixtec Ceramics (VUPA 33, 1987). Ron exploró una casa del Clásico en Yucuñudahui, reportada en 1984 en su libro The Mixtecs in Ancient and Colonial Times. Las monografias VUPA de Spores y Lind documentan el trabajo pionero de Ron y ya son obras clásicas de la literatura arqueológica de la Mixteca.

En 1970, Flannery y sus ayudantes visitamos a Ron y su grupo en Yucuita y Chachoapan para conocer los trabajos nuevos. Habían encontrado en el fondo de un pozo profundo (N203K) en Yucuita cerámica similar a la de la fase Tierras Largas, recientemente definida en el valle de Oaxaca. Nos sorprendió debido a que Kent pensaba que la altura del valle de Nochixtlán y el clima relativamente más frío que el del valle de Oaxaca no posibilitarían el descubrimiento de material tan temprano. Después aparecieron otros sitios tempranos en la Mixteca Alta y ahora sabemos que hubo una gran ocupación, por lo menos en el valle de Nochixtlán y sus alrededores, de aldeas del Preclásico Temprano con agricultura basada en el maíz. En otra ocasión, con Ron encontré una punta tipo Coxcatlán en la ladera este de la cima de Yucuñudahui. Fue la primera punta arcaica que habíamos visto en la Mixteca, similar a dos puntas encontradas por Flannery en Cueva Blanca y unas por MacNeish en el valle de Tehuacán. Unos años más tarde, Bruce Byland y John Pohl encontraron otra en su recorrido de superficie por el municipio de Jaltepec. Las semejanzas entre las puntas tipo Coxcatlán nos daban la idea de que los grupos arcaicos se movían en todo el territorio - el valle de Tehuacán, la Mixteca, el valle de Oaxaca y más allá- en vez de estar restringidos a un subvalle o a una región pequeña. Probablemente eran hablantes de proto-otomangue y sus descendientes, unos siglos más tarde, vivían en aldeas sedentarias y hablaban diversas lenguas otomangues.

En el verano de 1971, Ron y su esposa Marianne vivían con sus hijos Lisa y Jonathan (este último hizo años después una tesis de maestría sobre la obsidiana en Oaxaca) en una casa en el Rancho San Felipe, en Oaxaca, donde Cicely y yo también alquilamos un departamento, al igual que otros arqueólogos y estudiantes. En septiembre de 1971, recién casados y sin trabajo, decidimos trasladarnos a vivir a Nashville, cerca de Ron y su familia y la música country que nos gustaba. Ron nos presentó a un amigo de la industria musical, Robert Ferguson, y a su esposa Martha, ella en parte nativa americana de la tribu cherokee. Bob era ejecutivo en RCA; nos indicó dónde podíamos escuchar música y nos llevó a una sesión de grabación.

En años subsecuentes Ron estuvo ocupado con la universidad, la enseñanza, sus investigaciones en archivos y las publicaciones de la serie VUPA. Dos artículos suyos que me parecieron especialmente valiosos en estos años son "Marital Alliance in the Political Integration of Mixtec Kingdoms" (American Anthropologist, 1974, núm. 76, pp. 297-311) y "La estratificación social en la antigua sociedad mixteca" (en P. Carrasco y J. Broda [eds.], La estratificación social en la Mesoamérica prehispánica, 1976). En ellos planteó modelos para interpretar la organización prehispánica en la Mixteca Alta. En 1990, Ron y yo volvimos a trabajar juntos. Él pasaba una temporada en Tlaxiaco y yo tuve un proyecto en Huamelulpan. Ron, su amiga Anita Downs y la estudiante Vilma Fialko me ayudaron en las excavaciones en la parte alta del Grupo de la Iglesia. Después, Ron continuó con los recorridos de superficie en el área de Tlaxiaco, donde registró muchos sitios desconocidos hasta entonces. En 2004 emprendió un nuevo proyecto en el sitio de Yucundaa, el Pueblo Viejo de Teposcolula, que promete arrojar información valiosa sobre el periodo de contacto entre los españoles y los mixtecos.

Hoy en día, la arqueología de la Mixteca ya no es tan sencilla como lo era hace 40 años; participan más especialistas y ha habido nuevos hallazgos, datos e interpretaciones. Como buen maestro, Ron ha creado, formado e inspirado a estudiantes y, al mismo tiempo, ha sabido valorar los resultados de sus estudios, sean o no compatibles con los suyos. Ron tuvo la suerte de encontrar un enfoque fascinante y complejo para su vida. Yo y otros que hemos trabajado con él también hemos tenido la fortuna de contar con un maestro de amplia y contagiosa dedicación. Le estamos agradecidos por habernos hecho conocer a la Mixteca, su gente, su arqueología, su historia y sus comunidades. Sabemos que es imposible abarcar todo, pero Ron nos ha enseñado que podemos intentarlo. 